\title{
Iso-alpha acids from hops (Humulus lupulus) inhibit hepatic steatosis, inflammation, and fibrosis
}

\author{
Abdo Mahli ${ }^{1,2} \cdot$ Andreas Koch $^{1,2} \cdot \mathrm{Kim}$ Fresse $^{1} \cdot$ Tobias Schiergens $^{3} \cdot$ Wolfgang Erwin Thasler $^{3} \cdot$ \\ Christina Schönberger ${ }^{4} \cdot$ Ina Bergheim ${ }^{5}$ - Anja Bosserhoff ${ }^{1}$ - Claus Hellerbrand ${ }^{1,2}$
}

Received: 7 February 2018 / Revised: 8 June 2018 / Accepted: 24 June 2018 / Published online: 8 August 2018

(c) United States \& Canadian Academy of Pathology 2018

\begin{abstract}
Non-alcoholic fatty liver disease (NAFLD) is considered to be the hepatic manifestation of the metabolic syndrome. Isoalpha acids (IAAs), hop-derived bitter compounds in beer, have been shown to beneficially affect different components of the metabolic syndrome such as insulin resistance and dyslipidemia. However, IAAs have not yet been studied in the context of chronic liver disease. Here we analyzed the effect of IAA on the pathogenesis of NAFLD. Once, we applied IAA to mice in combination with a NAFLD-inducing Western-type diet (WTD), and observed that IAA significantly inhibited WTDinduced body weight gain, glucose intolerance, and hepatic steatosis. Fitting to this, IAA dose-dependently inhibited cellular lipid accumulation in primary human hepatocytes (PHH) in vitro. Reduced expression of PPAR-gamma and key enzymes of lipid synthesis as well as increased expression of PPAR-alpha, indicative for increased lipid combustion, were identified as underlying mechanisms of reduced hepatocellular steatosis in vitro and in vivo. Analysis of hepatic HMOX1 expression indicated reduced oxidative stress in IAA-treated mice, which was paralleled by reduced activation of the JNK pathway and pro-inflammatory gene expression and immune cell infiltration. Furthermore, IAA reduced hepatic stellate cell (HSC) activation and pro-fibrogenic gene expression. Similarly, IAA also dose-dependently reduced oxidative stress and JNK activation in steatotic PHH, inhibited HSC activation, and reduced proliferation and pro-fibrogenic gene expression in already activated HSC in vitro. In conclusion, IAAs inhibit different pathophysiological steps of disease progression in NAFLD. Together with previous studies, which demonstrated the safety of even long-term application of IAA in humans, our data suggest IAA as promising therapeutic agent for the prevention and treatment of (non)alcoholic (fatty) liver disease.
\end{abstract}

Electronic supplementary material The online version of this article (https://doi.org/10.1038/s41374-018-0112-x) contains supplementary material, which is available to authorized users.

Claus Hellerbrand

claus.hellerbrand@fau.de

1 Institute of Biochemistry (Emil-Fischer Zentrum), FriedrichAlexander University Erlangen-Nürnberg, Erlangen, Germany

2 Department of Internal Medicine I, University Hospital Regensburg, Regensburg, Germany

3 Biobank o.b. HTCR, Department of General Visceral and Transplantation Surgery, Ludwig-Maximilians-University Munich, Munich, Germany

4 Joh Barth \& Sohn GmbH \& Co. KG, Nürnberg, Germany

5 Department of Nutritional Sciences, Molecular Nutritional Science, University of Vienna, Vienna, Austria

\section{Introduction}

Non-alcoholic fatty liver disease (NAFLD) is recognized as the most common cause of liver enzyme elevations in Western countries [1]. In most cases, NAFLD is associated with obesity and insulin resistance, and therefore, is regarded as the hepatic manifestation of the metabolic syndrome. NAFLD encompasses a wide range of pathological conditions. Simple steatosis can progress to (nonalcoholic) steatohepatitis (NASH). In its advanced form, NASH is characterized by necroinflammation and progressive fibrosis [2]. The latter can progress to cirrhosis, and today, NASH is believed to account for a large fraction, if not entirely for what was previously termed "cryptogenic cirrhosis". Cirrhosis is the main risk factor for hepatocellular carcinoma (HCC), but is increasingly recognized that also simple steatosis without advanced inflammation and fibrosis significantly increases the HCC risk [3]. 
Hepatocellular fat accumulation is the first pathological step in NAFLD. Hepatic steatosis reflects a disordered lipid metabolism, and insulin resistance and excessive fatty acid (FA) influx to the liver are important contributing factors. Steatosis is also associated with lipotoxicity and (oxidative) cellular stress, which result in hepatocellular injury. Hepatic inflammation is frequently triggered by a variety of signals such as pro-inflammatory cytokines and chemokines, released by injured hepatocytes. Furthermore, sustained inflammation and hepatocellular injury are major triggers of hepatic fibrosis $[4,5]$.

Hepatic stellate cells (HSCs) represent the central mediators of hepatic fibrosis in chronic liver disease, including NAFLD. They are resident retinoid-storing cells located in the perisinusoidal space of Disse [6]. In response to various stimuli during hepatic injury, quiescent HSCs get activated and transform to highly proliferative myofibroblast-like cells, which are the cellular source of excessive extracellular matrix expression and deposition in chronic liver disease. Therefore, the activation of HSC is considered as a key event of hepatic fibrosis [7].

Until now, there is no established medical therapy for the prevention or treatment of NAFLD. It remains that the best available treatment is instructing patients to follow a reasonable dietary plan and a tailored exercise program. Thus, novel forms of therapies affecting individual pathological steps in NAFLD progression are urgently needed.

Iso-alpha acids (IAAs) are the main predominant bitter acids in beer derived from hops. They are constituted from humulones through the thermal isomerization during the brewing process (wort boiling with hop), thereby providing beer with its typical bitter taste and foam stability [8]. Recent studies showed that IAAs exhibit beneficial effects on different components of the metabolic syndrome, such as modulating the blood lipid status, reducing the metabolic endotoxemia, improving hyperglycemia, and decreasing visceral as well total body fat [9-14]. Moreover, it has been shown that IAAs have anti-inflammatory effects in models of different diseases such as Alzheimer's disease, osteoarthritis, cartilage degradation, and lipopolysaccharide-mediated inflammation [15-20]. These effects were attributed to reduced levels of reactive oxygen species (ROS) and pro-inflammatory cytokines and lower prostaglandin E2 formation mediated by specific inhibition of Cox2 and the GSK-3/NFkB pathway [15-20].

So far, effects of IAA on the progression of NAFLD have not been analyzed. In general and up to date, no study has systemically addressed the effects of IAA on different pathological stages of chronic liver disease.

The aim of this study was to evaluate the effect of a defined extract of IAAs in a dietary NAFLD model in mice. Furthermore, we performed in vitro experiments in which we investigated the effects of IAA on lipid accumulation and proinflammatory gene expression in $\mathrm{PHH}$ as well as on the activation and pro-fibrogenic phenotype of HSC. We found that IAA inhibits steatosis, inflammation, and fibrosis, i.e., the critical steps in NAFLD development and progression.

\section{Materials and methods}

\section{Cells and reagents}

Isolation and culture of primary human hepatocytes (PHH) and HSCs were performed as described [21-23]. Isohop $(30 \% \mathrm{v} / \mathrm{v})$ or freeze-dried potassium salt of isohumulone $(76.6 \% \mathrm{~m} / \mathrm{m})$ were provided by Joh Barth \& Sohn GmbH \& Co. KG (Nuremberg, Germany). Cells were incubated with IAA in concentrations and time intervals as indicated. In some conditions, cells were pre-treated with a specific peroxisome proliferator-activated receptor-alpha (PPAR- $\alpha$ ) inhibitor $(10 \mu \mathrm{M}$, GW 6471; Cay11697-1; Biomol GmbH, Hamburg) as indicated.

\section{Animals and animal models}

Ten-week-old C57BL/6 mice (Charles River Laboratories (Sulzfeld, Germany) were divided into three groups (7-8 mice/group) and were fed either with control diet or a Western-type diet (WTD) with or without supplementation of $0.5 \% \mathrm{w} / \mathrm{w}$ freeze-dried potassium salt of isohumulone (equivalent to $500 \mathrm{mg} / \mathrm{kg}$ body weight (BW)). Chows were prepared by Ssniff (Soest, Germany). The dose of IAA was chosen based on previous in vivo studies [11, 24, 25]. Oral glucose tolerance test was performed as described [26] 12 weeks after start of feeding of the experimental diets. After 15 weeks, mice were sacrificed and liver tissue and blood samples were collected for further analysis.

\section{Analysis of cellular lipid content}

Cellular lipid droplets were visualized by Oil Red O staining [27]. Total cellular triglyceride levels were quantified as described [28].

\section{RNA expression analysis}

RNA isolation from cultured cells and tissues and reverse transcription were performed as described [29]. Quantitative real-time PCR (RT-PCR) was performed applying LightCycler technology (Roche) using specific primer pairs as described in ref. [30].

\section{Protein analysis}

Protein extraction and Western blotting were performed as described [31] applying anti-rabbit antibodies against 
phospho-JNK (\#9251), phospho-Jun (\#3270), and c-Jun (\#9165) all from Cell Signaling Technology (Danvers, MA, USA; all diluted 1:1,000). Furthermore, antibodies against alpha smooth muscle actin ( $\alpha$-SMA) (ab5694 from Abcam, Cambridge, UK; 1:1,000) or actin (MAB1501 from Merck Millipore, Billerica, MA, USA; 1:3000) were applied. For densitometry analysis, Licor Image Studio Lite software (LI-COR Biotechnology; Homburg, Germany) was used.

Adipokines in murine serum samples were measured by enzyme-linked immunosorbent assay (ELISA) analysis using the adiponectin and leptin DuoSet ELISA Development kits from R\&D Systems (Minneapolis, MN, USA) according to the manufacturer's instructions.

\section{(Immuno)histological analyses}

For histological analysis murine liver tissue specimens were fixed for $24 \mathrm{~h}$ in $4 \%$ formalin at room temperature, dehydrated by graded ethanol, and embedded in paraffin. Tissue sections (thickness $5 \mu \mathrm{m}$ ) were deparaffinized with xylene and stained with hematoxylin and eosin. Immunohistochemical staining was performed using anti-CD3 C7930 antibody (1:500; Sigma-Aldrich) as described [26]. Hepatic immunohistochemical staining for phosphorylated c-Jun was performed as described [26]. The number of CD3positive hepatocytes was counted in four randomly selected areas on each section.

\section{Analysis of cell proliferation assay}

Colorimetric XTT assay (Roche Diagnostics, Mannheim, Germany) was used according to the manufacturer's instructions for quantification of cell proliferation.

\section{Statistical analysis}

Results are expressed as mean \pm SD. Comparisons between groups were performed using the unpaired Student's $t$-test or one-way analysis of variance, if appropriate. A $p$ value $<$ 0.05 was considered statistically significant. All calculations were carried out using the statistical computer package GraphPad Prism version 4.00 for Windows (GraphPad Software, San Diego, USA).

\section{Results}

\section{Effect of isomerized alpha acids on obesity and other elements of the metabolic syndrome in mice fed with a WTD}

For this study, we used a defined extract of isomerized alpha acids (powdered form of potassium salts of isohumulone, with an analysis of $76.6 \%$ IAA content). Previous studies have shown that isomerized alpha acids are very stable [32, 33], and thus, it can be assumed that also in the experiments performed in this study, the composition of individual humulones remained invariable. In the following, IAA is used as abbreviation for this IAA extract, which was used throughout the in vivo and in vitro experiments.

First, IAA effects were studied in mice fed with a WTD [26]. We have previously shown that mice fed with this diet develop key components of the metabolic syndrome and their liver pathology mimics critical pathological steps of human NAFLD [26, 34]. This WTD was applied either alone or supplemented with $0.5 \%$ IAA w/w for 15 weeks. Mice receiving standard chow served as control. No significant differences were found between treatment groups regarding food and fluid intake throughout the study (data not shown). Feeding the WTD led to significant body weight gain over time compared with mice fed with standard control chow (Fig. 1a). After 15 weeks WTD-fed mice appeared obese and showed a marked enlargement of both visceral and subcutaneous adipose tissue (Fig. 1a, b). Serum concentration of leptin was significantly elevated, whereas circulating adiponectin levels were decreased in WTD-fed compared with control mice (Fig. 1c, d) mimicking an adipokine serum profile associated with obesity in humans [35]. Furthermore, glucose tolerance was impaired in WTDfed mice (Fig. 1e) pointing to manifest insulin resistance. In contrast, IAA application completely prevented WTDinduced weight gain (Fig. 1a) and expansion of adipose tissue was less pronounced (Fig. 1b). Furthermore, serum leptin levels were significantly lower and adiponectin levels significantly higher in mice receiving IAA together with the WTD compared to mice fed with WTD only (Fig. 1c, d). Similarly, glucose tolerance was less impaired in the IAA group compared with mice that were fed with WTD without IAA supplementation (Fig. 1e). In summary, IAA application significantly ameliorated WTD-induced body weight gain, expansion of adipose tissue and insulin resistance.

\section{Effect of the IAA on WTD-induced hepatic steatosis and lipid metabolism}

Next, we wanted to analyze the impact of IAA on WTDinduced changes in hepatic lipid accumulation and metabolism. WTD-fed mice revealed remarkable hepatomegaly (Fig. 2a) and a significantly increased liver weight (Fig. 2b) compared with control mice. These WTD-induced alterations were almost completely blunted in the IAA + WTD group (Fig. 2a, b). Macroscopic liver appearance in the WTD group was indicative for hepatic steatosis (Fig. 2a), and assessment of hepatic triglycerides confirmed significantly elevated levels in WTD-fed mice (Fig. 2c). Histological analysis showed mixed micro- and macrovesicular 
a

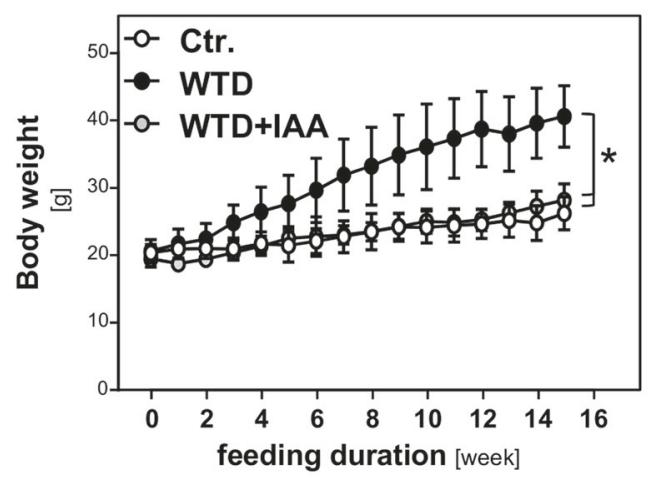

b

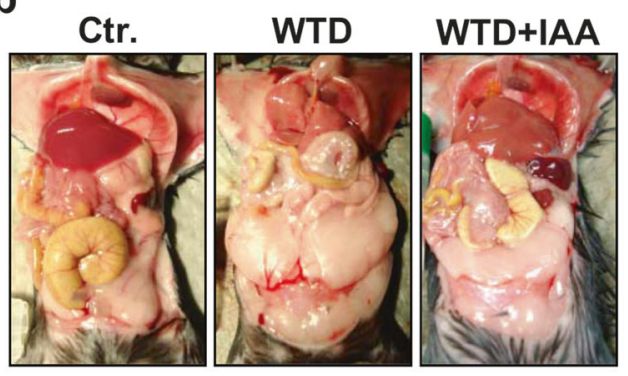

e

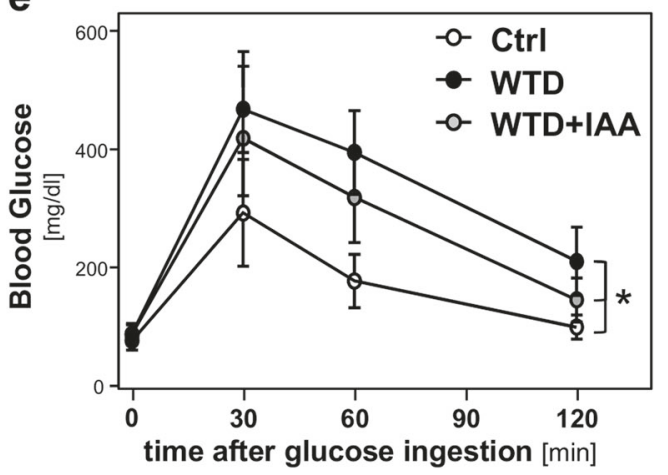

Fig. 1 Effect of IAA on obesity and other elements of metabolic syndrome. Mice were fed either with control diet (Ctr.) or a Westerntype diet (WTD) with (WTD + IAA) or without (WTD) supplementation of $0.5 \% \mathrm{w} / \mathrm{w}$ of iso-alpha acids (IAAs) for 15 weeks. a Body weight during the experiment (left panel) and representative

steatosis (Fig. 2a). In both biochemical and histological analysis WTD-induced hepatic steatosis was significantly reduced in IAA + WTD group compared to the WTD group (Fig. 2a, c).

In search for the underlying mechanisms of IAA effect on hepatic steatosis, we analyzed the gene expression alterations of several key factors known to have a critical role in hepatic lipid metabolism and to be also frequently altered in NAFLD patients (reviewed in ref. [36]). PPAR- $\alpha$ is a strong regulator of hepatic energy combustion, while $\operatorname{PPAR} \gamma$ has been shown to induce lipid synthesis and storage. Expression of both transcription factors was enhanced in WTD-fed mice (Fig. 2d, e) similar as described in human pictures of mice after 15 weeks of feeding (right panel). b Laparotomy showing massively increased visceral and subcutaneous white adipose tissue depots of WTD compared with control or WTD + IAA mice. Serum levels of $\mathbf{c}$ leptin and $\mathbf{d}$ adiponectin. e Glucose levels derived from a glucose tolerance test $(* p<0.05)$

NASH [37] indicative for a marked activation of the overall hepatic lipid metabolism. In the IAA + WTD group, PPAR $\gamma$ expression was significantly reduced compared with the WTD group while PPAR $\alpha$ expression levels did not significantly differ (Fig. 2d, e). Fitting to this, WTD diet induced a marked increase of FA synthase (FASN) and stearoyl-CoA desaturase 1 (SCD1), both central regulators of de novo lipogenesis (Fig. 2f, g). IAA application significantly reduced the WTD-induced increase of FASN and SCD1 expression (Fig. 2f, g). Furthermore, WTD feeding led to a significant upregulation of the expression of mitochondrial carnitine palmitoyltransferase I (CPT1) and peroxisomal acyl-CoA oxidase $1(A C O X 1)$, genes involved in 
a

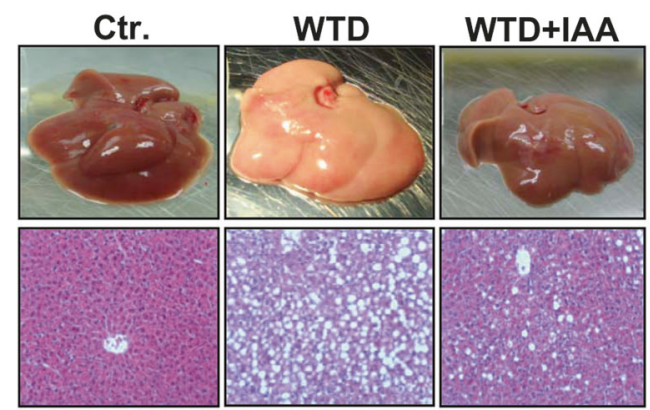

d
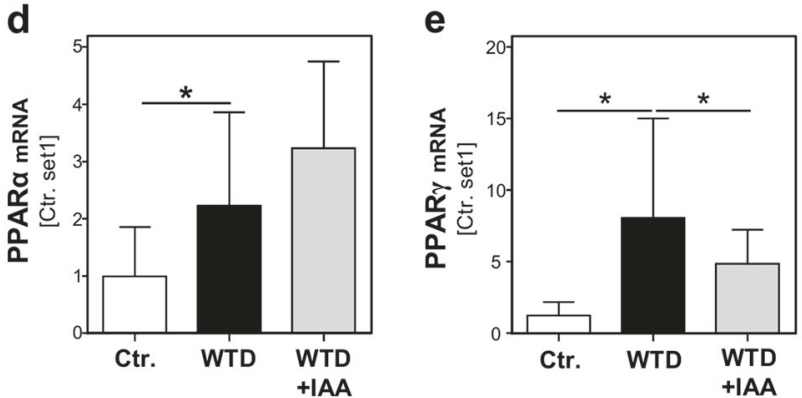

h

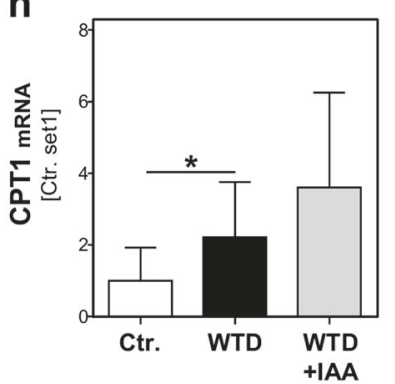

b

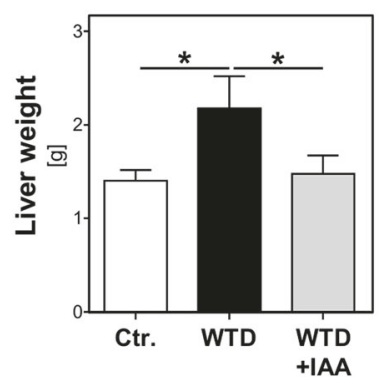

f

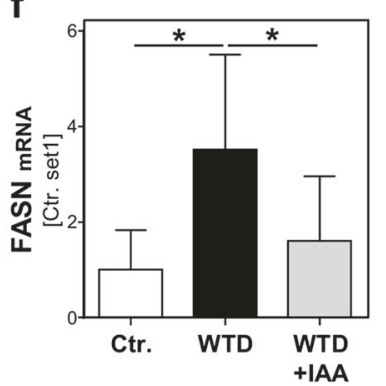

C

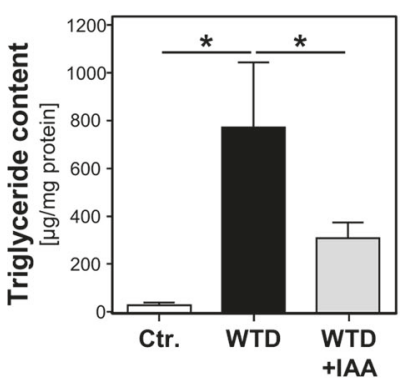

g

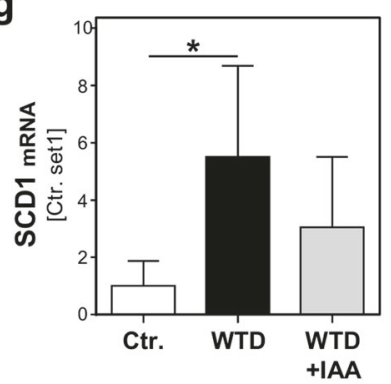

Fig. 2 Effect of the IAA on WTD-induced hepatic steatosis and lipid metabolism. Mice were fed either with control diet (Ctr.) or a Westerntype diet (WTD) with (WTD + IAA) or without (WTD) supplementation of $0.5 \% \mathrm{w} / \mathrm{w}$ of iso-alpha acids (IAAs) for 15 weeks. a Macroscopic images (upper pictures) and histological staining

FA oxidation, compared to control mice (Fig. 2h, i). These upregulation of gene expression was further increased by IAA application but differences did not reach the level of significance (Fig. 2h, i). Beta-oxidation of FA is causing formation of ROS, and in the liver of WTD-fed mice expression of HMOX1, a marker for oxidative stress, was significantly increased compared to the control group (Fig. 2j). Interestingly, HMOX1 expression in the WTD + IAA group did not differ from controls (Fig. 2j), indicating antioxidant properties of IAA.

Together these data show that IAA application does not only prevent enlargement of adipose tissue but also hepatic steatosis and indicate an inhibitory effect on hepatic de novo lipogenesis and metabolism. Dual action as PPAR $\alpha$ agonist and PPAR $\gamma$ antagonist appears as underlying mechanisms of the reduced hepatic lipid accumulation.

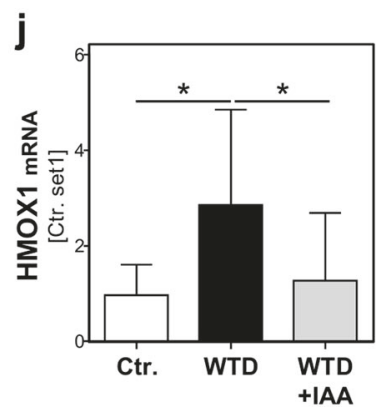

(hematoxylin/eosin; lower pictures). b Liver weight. c Hepatic triglyceride (TG) content normalized to total hepatic protein. Hepatic mRNA levels of $\mathbf{d}$ PPAR- $\alpha, \mathbf{e}$ PPAR- $\gamma, \mathbf{f}$ FASN, $\mathbf{g}$ SCD1, $\mathbf{h}$ CPT1, $\mathbf{i}$ ACOX1, and $\mathbf{j}$ HMOX1 analyzed by quantitative RT-PCR $(* p<0.05)$

\section{Effect of the IAA on lipid accumulation and metabolism in PHH}

Impaired insulin resistance and pathological adipokine levels are known modulators of hepatic lipid metabolisms. To dissect indirect IAA effects on hepatic steatosis from direct IAA-mediated effect on hepatocytes we applied an in vitro model of hepatocellular lipid accumulation in PHH that we have previously described [27]. In this model, lipid accumulation in PHH was induced by incubation with FAs ( $0.3 \mathrm{mM}$ of oleate) for $24 \mathrm{~h}$. We have previous shown that hepatocellular steatosis induced by this oleate concentration led to pathological alterations similar to those found in hepatic tissue of NAFLD patients [27, 35]. IAAs were applied in doses up to $20 \mu \mathrm{g} / \mathrm{ml}$. Previous studies have shown that these concentrations are reached in the serum of 
a

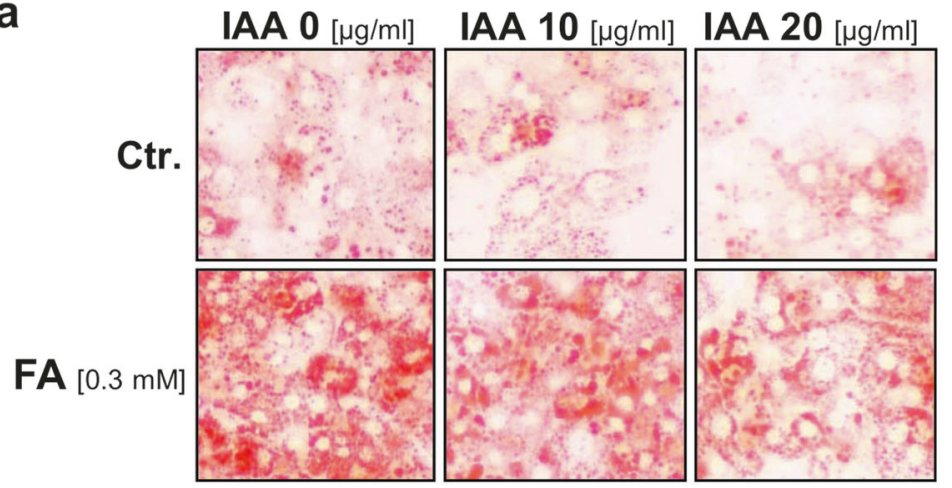

C

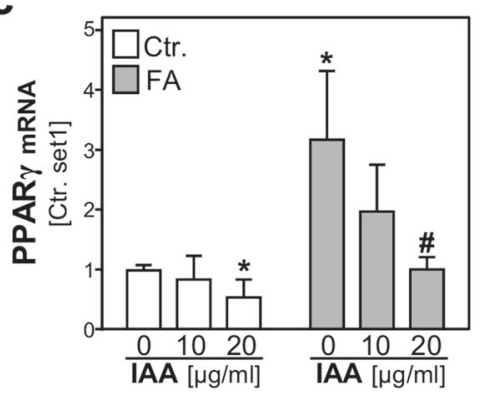

e

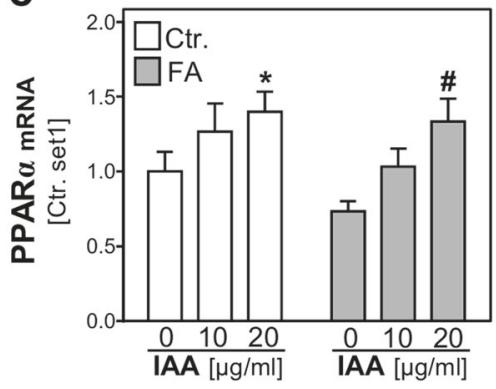

d

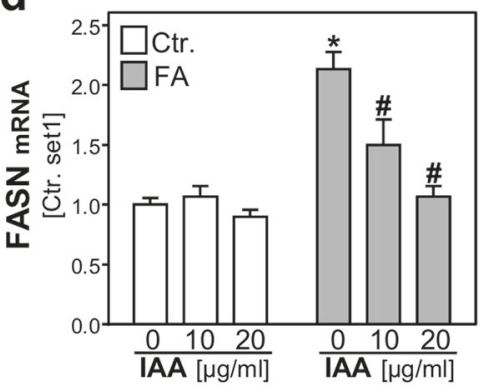

f

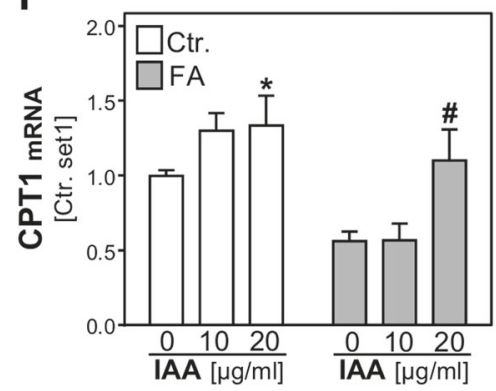

b
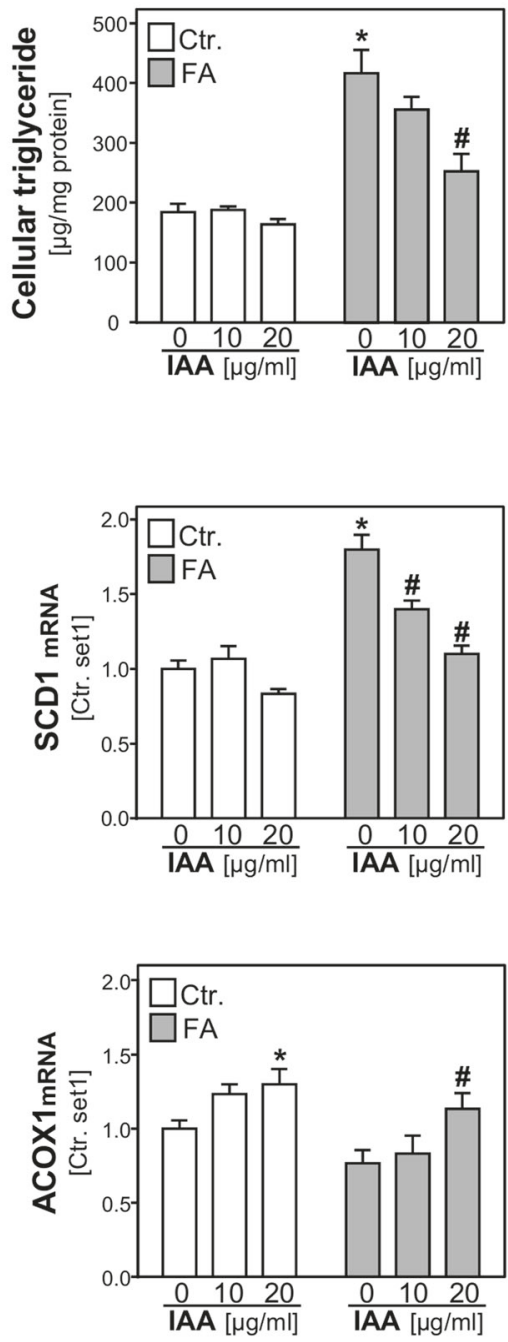

Fig. 3 Effect of the IAA on lipid accumulation and metabolism in primary human hepatocytes $(\mathrm{PHH})$. $\mathrm{PHH}$ were treated with IAA and fatty acids (FAs) ( $0.3 \mathrm{mM}$ of oleate) for $24 \mathrm{~h}$. a Microscopic images of Oil Red O staining. b Hepatocellular triglyceride (TG) content normalized to cellular protein content. Cellular mRNA levels of $\mathbf{c}$ PPAR $\gamma, \mathbf{d}$ FASN and SCD1, e PPAR $\alpha$, and $\mathbf{f ~ C P T 1 ~ a n d ~ A C O X 1 ~} 1{ }^{*} p<$ 0.05 compared to control; ${ }^{\#} p<0.05$ compared to FA alone)

PPAR $\alpha$ expression are of importance for the influence of IAA on lipid metabolisms. In summary, these in vitro data show that IAAs directly affect lipid accumulation and metabolism in hepatocytes independent of systemic effect on other components of the metabolic syndrome.

\section{Effect of IAA on steatosis-induced hepatic inflammation}

Next, we wanted to analyze the effects of IAA on WTDinduced hepatic inflammation, which constitutes the second pathological step in NAFLD progression. WTD feeding caused markedly increased hepatic expression levels of the cytokine (CXCL1), the adhesion molecule (ICAM-1), tumor necrosis factor and interleukin-1 (Fig. 4a). These pro(supplementary Figure 2), indicating that IAA effects on 
a
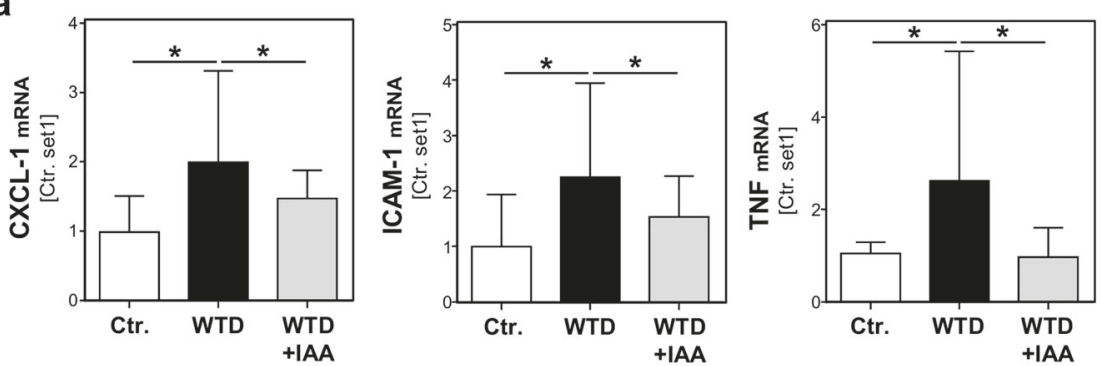

b

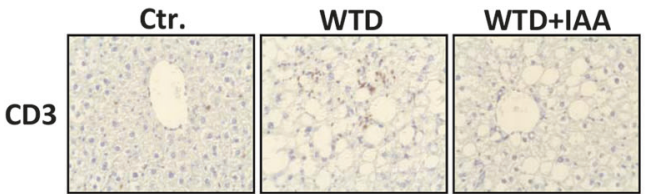

d

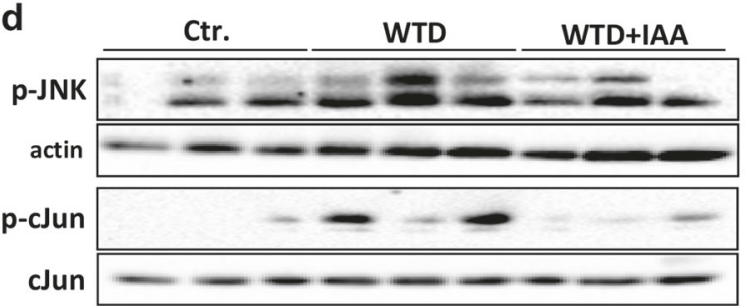

e
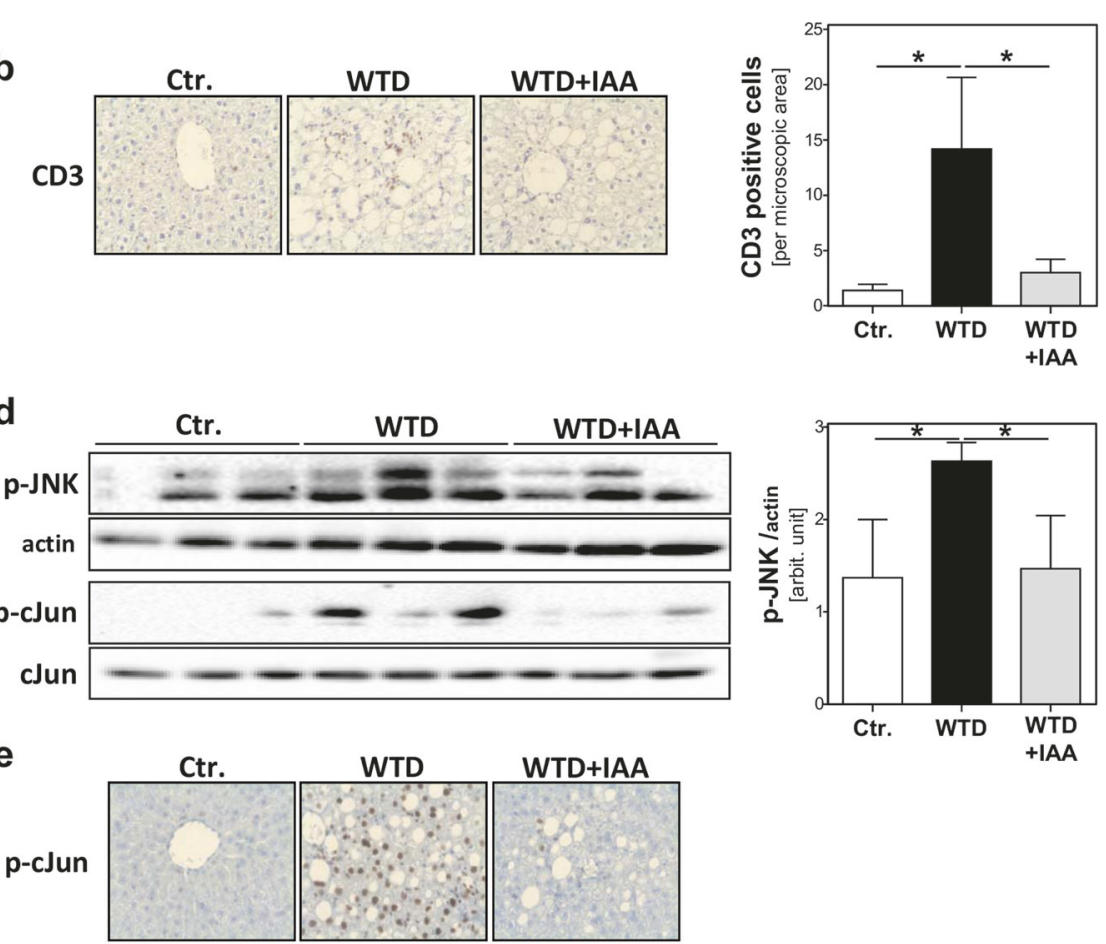

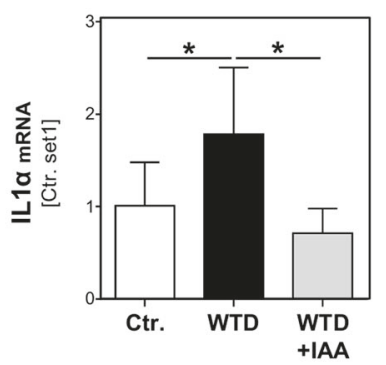

C
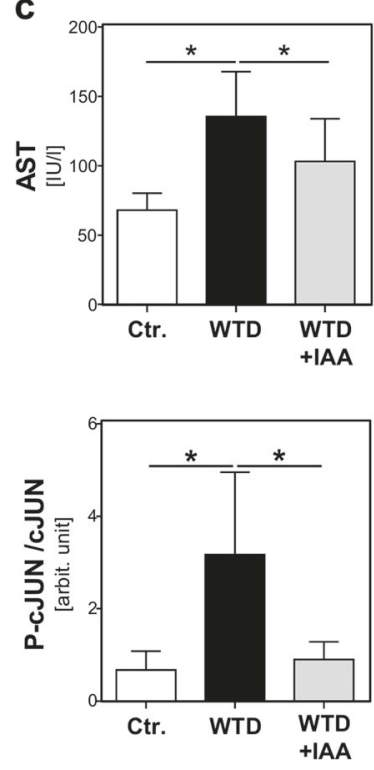

f
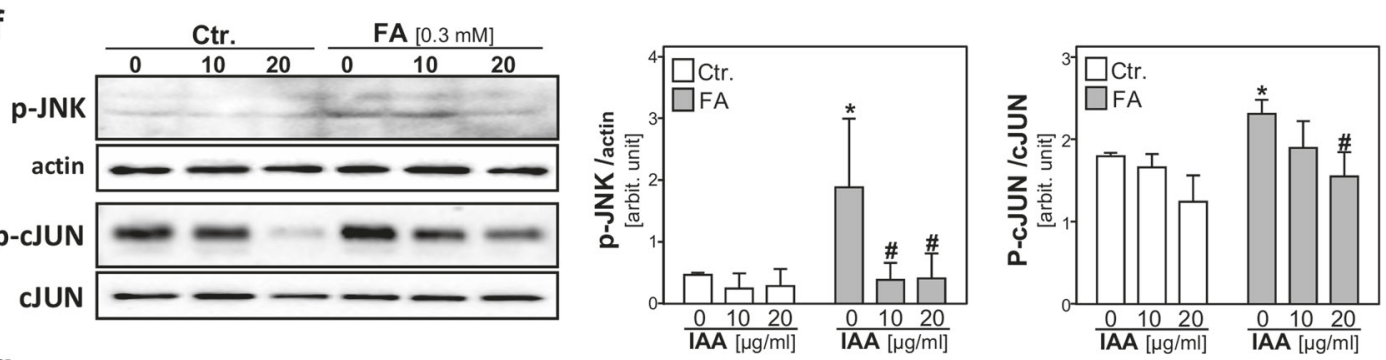

g
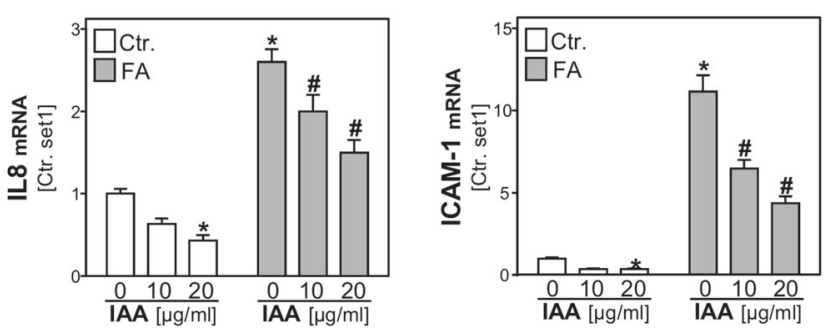

inflammatory factors have been shown to play a crucial role in NASH development and progression [38]. Notably, the WTD-induced expression of these pro-inflammatory factors was completely blunted by IAA application (Fig. 4a). CD3 immunohitochemical analysis confirmed hepatic infiltration with inflammatory cells in WTD-fed mice, which was 
Fig. 4 Effect of IAA on steatosis-induced hepatic inflammation. Mice were fed with control diet (Ctr.) or a Western-type diet (WTD) with (WTD + IAA) or without (WTD) supplementation of $0.5 \% \mathrm{w} / \mathrm{w}$ of iso-alpha acids (IAAs) for 15 weeks. a CXCL1, ICAM-1, TNF, and IL-1 $\alpha$ mRNA levels analyzed by quantitative RT-PCR. b Microscopic images of CD3 immunohistochemical staining of liver tissue (left panel) and quantification of CD3-stained cells (right panel). c Serum aspartate aminotransferase (AST) levels). d Western blot analysis of pJNK and p-cJUN protein levels (left panel); actin served as control for loading adjustment (left panels); densitometric analysis of the corresponding blots (right panels). e Microscopic images of p-cJUN immunohistochemical staining of liver tissue. $(* p<0.05)$. Primary human hepatocytes $(\mathrm{PHH})$ were pre-treated with IAA (doses up to $20 \mu \mathrm{g} / \mathrm{ml}$ ) for $24 \mathrm{~h}$. Subsequently, cells were co-incubated with fatty acids (FA) ( $0.3 \mathrm{mM}$ of oleate) for $24 \mathrm{~h}$. f Western blot analysis of pJNK and p-cJUN protein levels; actin served as control for loading adjustment (left panels); densitometric analysis of the corresponding blots (right panels). g Cellular IL-8 and ICAM-1 mRNA levels analyzed by quantitative RT-PCR $\left(* p<0.05\right.$ compared to control; ${ }^{*} p<$ 0.05 compared to FA alone)

almost completely inhibited by IAA application (Fig. 4b). In accordance, aspartate aminotransferase (Fig. 4c) and alanine aminotransferase (data not shown) serum levels, markers for hepatic injury, were significantly increased in the WTD group but they were significantly reduced in WTD + IAA. We and others have shown that sustained activation of the JNK pathway in steatotic hepatocytes is a central mediator of hepatic inflammation in fatty liver disease [26, 39]. Also here, JNK and cJUN phosphorylation were significantly induced in the liver of WTD-fed mice, and this increased activation of the JNK pathway was completely blunted in mice that received IAA in addition to the WTD (Fig. 4d). Immunohistochemical analysis of phosphorylated cJUN expression confirmed nuclear translocation into hepatocytes in the WTD group, which was almost absent in the WTD + IAA group (Fig. 4e).

Besides hepatic steatosis, also obesity-associated pathological alterations such as altered adipokine levels have a major impact on hepatic inflammation [40]. To investigate whether IAAs exhibit also direct antiinflammatory effects on steatotic hepatocytes, we applied again the in vitro model of hepatocellular lipid accumulation. Lipid accumulation caused a significant increase of JNK and cJun phosphorylation, which was completely blunted by IAA (Fig. 4f). Similarly, steatosis induced expression of pro-inflammatory genes was dosedependently reduced by IAA incubation in hepatocytes (Fig. 4g). Notably, also in control hepatocytes c-Jun phosphorylation and pro-inflammatory gene expression were significantly reduced by IAA (Fig. 4f, g).

Together, these data indicate that IAAs inhibit steatosisinduced hepatic inflammation and that this is (also) caused by inhibitory effects on the JNK pathway and proinflammatory gene expression in hepatocytes.

\section{Effect of IAA on steatosis-induced hepatic fibrosis and HSC activation}

Sustained hepatocellular injury and inflammation can lead to hepatic fibrosis. The activation of HSCs is the key event of hepatic fibrosis [6]. Also in NAFLD, activated HSCs are the major cellular source of extracellular matrix deposition [41]. In the murine NASH model, liver histology did not yet show prominent hepatic fibrosis in mice fed with WTDinducing NASH (data not shown). However, WTD-fed mice revealed significantly heightened $\mathrm{mRNA}$ and protein levels of hepatic $\alpha$-SMA (Fig. 5a, b), an established marker of activated HSC [41]. This was accompanied by a distinct pro-fibrogenic transcriptional profile, characterized by significantly elevated hepatic levels of transforming growth factor beta-1 (TGF 1 1), tissue inhibitor of metalloproteinases-1, and matrix metalloproteinase-1, pro-fibrogenic genes highly expressed in activated HSC [42] (Fig. 5c). WTD-induced $\alpha$-SMA and pro-fibrogenic gene expressions were completely blunted in the WTD + IAA group. Accordingly, collagen type I (COL1Al) gene transcription was significantly elevated in WTD-fed compared to control mice, while COL1A1 expression levels in the WTD + IAA group did not differ from controls (Fig. 5d). Together these data clearly showed an anti-fibrogenic effect of IAA in the murine NASH model.

However, it was unclear, whether IAA directly affect HSC or whether IAA exhibit their anti-fibrotic effects via indirect (inhibitory) mechanisms such as via modulation of hepatic steatosis and inflammation, which are critical inducers of fibrosis in NAFLD [43].

To investigate whether IAA exhibit direct anti-fibrogenic effects, we first analyzed the IAA effects on the in vitro activation process of HSC. Two days after isolation, human HSCs were exposed to non-toxic concentrations of IAA at three different doses $(5,10$, and $20 \mu \mathrm{g} / \mathrm{ml})$ for 7 days (supplementary Figure 1a). Subsequently, mRNA expression of two established markers of HSC activation, namely COL1A1 and $\alpha$-SMA, were determined by quantitative RTPCR analysis. Treatment with IAA significantly reduced the expression of both markers compared to control HSC (Fig. 5e) and also microscopic images indicate an inhibitory effect on the in vitro transforming process to activated myofibroblast-like cells (Fig. 5f). Once they are activated, the proliferation rate of HSC significantly increases, a mechanism that has been proposed to play a key role in the progression of fibrosis in chronic liver disease, including NAFLD. Notably, incubation of in vitro-activated HSC with non-toxic concentrations of IAA (supplementary Figure $1 \mathrm{~b})$ led to dose-dependent reduction of cyclin D1 expression (Fig. 5g) and reduced proliferation (Fig. 5h). Moreover, we observed that treatment of activated HSC with IAA reduced the COL1A1 and TGF $\beta 1$ expression 

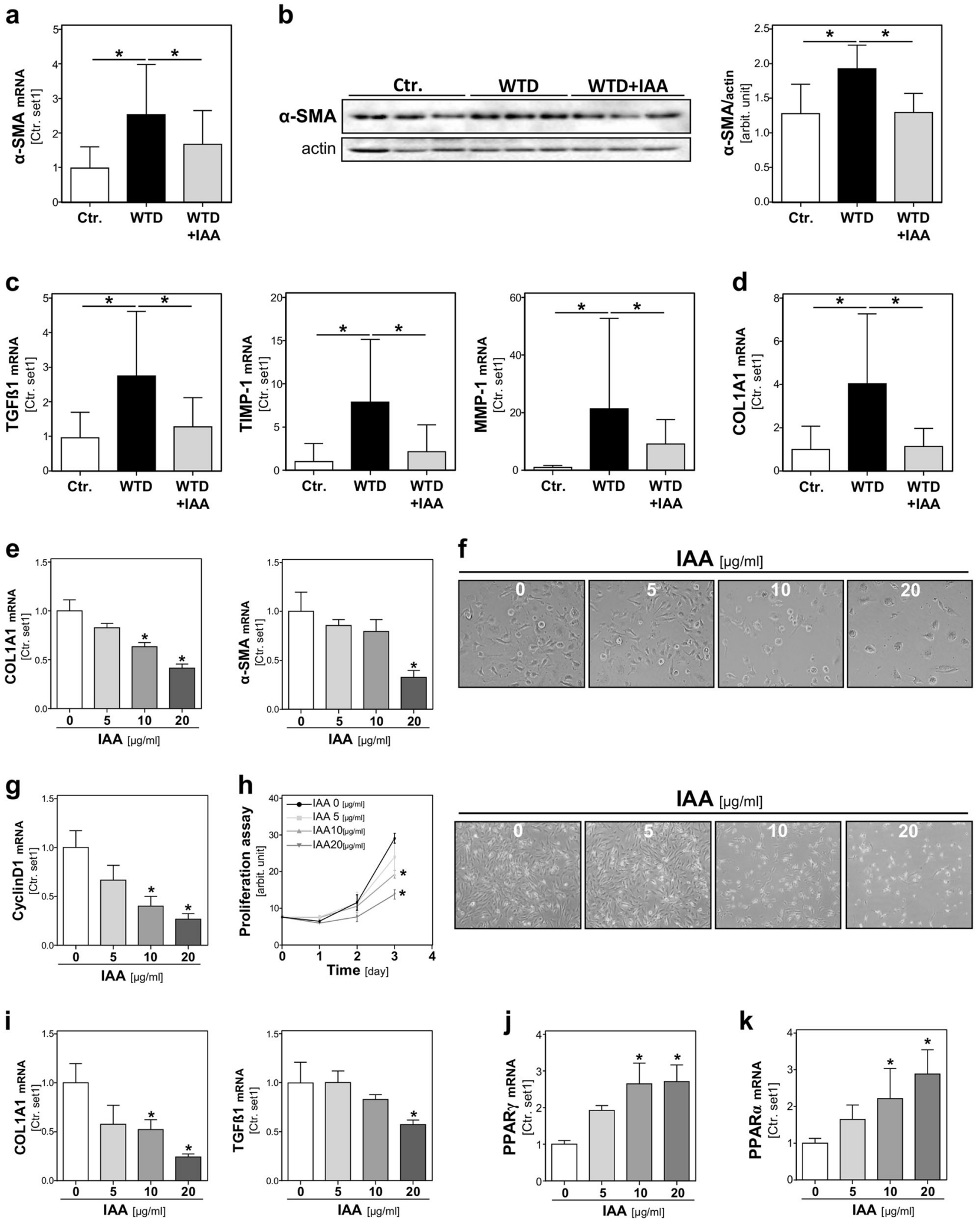

levels (Fig. 5i). Furthermore, we analyzed the effect of IAA on PPAR $\alpha$ and PPAR $\gamma$ expression in HSC. Similarly as in hepatocytes, IAA dose-dependently induced PPAR $\alpha$

expression in HSC (Fig. 5j). However, and in contrast to our observation in hepatocytes, incubation with IAA resulted also in a dose-dependent induction of PPAR $\gamma$ expression in 
Fig. 5 Effect of IAA on steatosis-induced hepatic fibrosis and hepatic stellate cell (HSC) activation. Mice were fed with control diet (Ctr.) or a Western-type diet (WTD) with (WTD + IAA) or without (WTD) supplementation of $0.5 \% \mathrm{w} / \mathrm{w}$ of iso-alpha acids (IAAs) for 15 weeks. a Hepatic $\alpha$-SMA mRNA levels analyzed by quantitative RT-PCR. b Western blot analysis of $\alpha$-SMA protein levels; actin served as control for loading adjustment (left panel); densitometric analysis of the corresponding blot (right panel). c Hepatic TGF $\beta 1$, TIMP-1, and MMP-1 mRNA levels analyzed by quantitative RT-PCR. d Hepatic COL1A1 mRNA levels analyzed by quantitative RT-PCR $\left({ }^{*} p<0.05\right)$. Human HSCs were activated by culturing on plastic dishes for 7 days with or without IAA treatment (doses up to $20 \mu \mathrm{g} / \mathrm{ml}$ ). e Cellular $\alpha$-SMA and COL1A1 mRNA levels analyzed by quantitative RT-PCR. f Microscopic phase-contrast images of freshly cultured HSC after 7 days of incubation with or without the indicated concentrations of IAA $\left({ }^{*} p<\right.$ 0.05 compared to $0 \mu \mathrm{g} / \mathrm{ml}$ IAA). Activated HSCs were treated with IAA for $24 \mathrm{~h}$. g Cellular cyclin D1 mRNA levels analyzed by quantitative RT-PCR. h Proliferation of HSCs assessed with the colorimetric XTT assay (left panel); phase-contrast images of activated HSC (right panel). Cellular mRNA levels of (i) COL1A1 and TGF $\beta 1$ as well as (j) PPAR $\gamma$ and $\mathbf{k}$ PPAR $\alpha$ analyzed by quantitative RT-PCR $\left({ }^{*} p<\right.$ 0.05 compared to $0 \mu \mathrm{g} / \mathrm{ml}$ IAA)

HSC (Fig. 5k). This is of interest, because it has been shown that activation of PPAR $\gamma$ in the HSCs has anti-fibrotic effects [44, 45], and thus, stimulatory effects on PPAR $\gamma$ may contribute to the observed anti-fibrogenic effects of IAA. In summary, IAA significantly inhibit hepatic fibrosis in a murine NASH model and in vitro data indicate that this anti-fibrogenic effect is (also) mediated via inhibitory effects on HSC activation and the pro-fibrogenic phenotype of already activated HSC, respectively.

\section{Discussion}

In this study, we characterized the effects of an IAA extract on different pathological steps of NAFLD in mice fed with a WTD, consisting of a high fat, cholesterol, and fructose content. It is increasingly recognized that besides lipid, also excessive cholesterol and fructose consumption significantly contribute to the development and progression of NAFLD. Importantly, we have previously shown that feeding this WTD to mice caused pathological changes in the liver that closely mimic liver pathology observed in patients with NAFLD [26, 35]. Today, this chronic liver disease is considered to be the hepatic manifestation of the metabolic syndrome, and it has been shown that improvement of individual components of the metabolic syndrome such as reduction of body weight or correction of insulin resistance or dyslipidemia has beneficial effects on NAFLD, too [46]. Previous studies have shown that IAAs have a beneficial effect on body fat mass, elevated blood glucose levels, elevated triglycerides associated with insulin resistance as well as hepatic steatosis in rodents fed with high-fat or atherogenic diets as well as genetic models of obesity and diabetes [9-13, 18, 24, 47, 48]. However, these studies have not systemically investigated the effects of IAA on the different pathological steps of WTD-induced chronic fatty liver disease.

In the present study, we have first shown that IAA application almost completely prevented the weight gain in WTD-fed mice. IAA supplementation led to a decrease in adipose tissue mass and improved glucose tolerance. Moreover, IAA caused in WTD-fed mice a normalization of the dysregulated levels of adipokines, which are implicated in the pathogenesis of metabolic syndrome [49]. Together, our data verified that IAA improved systemic components of metabolic syndrome also in mice fed with a WTD. Although significant, IAA effects of on individual components of metabolic syndrome quantitatively varied. For instance, weight gain was most effectively prevented while glucose tolerance was significantly improved but still impaired. This is in line with previous studies that analyzes the effect of isohumulone or hop extracts in models of obesity and diabetes. These studies also found the strongest IAA effect on body weight gain $[47,50]$. One may speculate that responsiveness of different tissues and cells or pathophysiological mechanisms, respectively, to IAA varies. Thus, it was important that we found that IAA supplementation also significantly inhibited hepatic steatosis in our model. Although previous studies showed that IAA can affect PPAR $\alpha$ and PPAR $\gamma$ [11-13, 24, 48, 50], the effects on steatotic liver tissue and specifically on hepatocytes were largely unknown. Moreover, it has to be noted that lipid accumulation in hepatocytes is driven by high circulating levels of FAs and insulin resistance. Therefore, it was critical to show whether the IAA-induced inhibition of hepatic steatosis is mediated (only) via (indirect) systemic effects or whether IAAs also directly affect lipid accumulation in hepatocytes. To address this question, we applied an in vitro model of hepatic steatosis on PHH. In this model, incubation with FAs complexed to albumin induced hepatocellular lipid accumulation, and we have previously shown that this very well reflects pathological changes observed in steatotic livers of NAFLD patients [35, 51]. Similar to in vivo results, IAA application almost completely inhibited the FA-induced lipid accumulation in $\mathrm{PHH}$ in vitro, indicating that in addition to systemic effects, IAA also directly affect hepatocellular steatosis. Hepatic steatosis is also associated with lipotoxicity and (oxidative) cellular stress which trigger the hepatocellular injury and inflammation. We and others have shown that hepatic steatosis causes JNK activation and that the activation of this pathway plays a critical role in steatosisinduced liver damage and inflammation [26, 52]. Notably, we observed that IAA significantly reduced steatosisinduced JNK activity in hepatocytes in vitro and in vivo. 
These findings indicate novel mechanisms by which IAAs inhibit steatosis-induced liver injury and inflammation. Both pathologies trigger hepatic fibrosis, the critical disease for morbidity and mortality in NAFLD. Importantly, we found that IAA treatment almost completely ameliorated the WTD-induced pro-fibrogenic gene expression and the activation of HSC. So far, anti-fibrogenic effects of IAA have not been described. Thus, it was unclear whether the inhibitory IAA effect on hepatic fibrosis only resulted from the beneficial IAA effects on hepatocellular injury and inflammation, or whether IAAs also directly affect HSC activation and fibrogenic gene expression. Similar as with regards to the IAA effect on hepatocellular lipid accumulation, we used an in vitro approach to address this question. Interestingly, IAA significantly reduced the activation of freshly isolated quiescent HSC as well as the pro-fibrogenic phenotype of already activated HSC, indicating a direct anti-fibrogenic effect of IAA on the key mediator cells of hepatic fibrosis.

Taken together, our study revealed that IAAs affect several critical pathological steps of NAFLD via different, in part cell type-specific mechanisms. Thus, IAA showed dual action as PPAR $\alpha$ agonist and PPAR $\gamma$ antagonist in hepatocytes, which appears as underlying mechanisms of reduced steatosis. In contrast, IAA induced PPAR $\gamma$ expression in HSC, and activation of PPAR $\gamma$ in HSC has been shown to have anti-fibrotic effects [44, 45]. Furthermore, IAAs inhibit in hepatocytes the steatosis-induced JNK/cJUN activation, a known driver of hepatic inflammation.

Currently, beer is the only nutritional source of IAA in humans. However, one has to note that no comparable IAA doses as applied in our mouse model can be achieved in humans by beer consumption. Based on calculation using allometric interspecies scaling [53] the mouse dose (500 $\mathrm{mg} / \mathrm{kg} \mathrm{BW}$ ) corresponds to a dose of approximately $42 \mathrm{mg} /$ $\mathrm{kg} \mathrm{BW}$ in humans. IAA concentration in beer varies between 15 and $100 \mathrm{mg} / \mathrm{l}$ [54]. Accordingly, a consumption of more than 1001 beer would be required to achieve the equivalent dose that was applied in the present study to mice. Still, further studies are required to assess the effective IAA doses in humans. Interestingly, results from epidemiological data as well as animal studies suggest that different alcoholic beverages, e.g., hard spirits and fermented beverages might differ in regards to their potency to harm the liver [55]. Kanuri et al. [55] reported that acute intake of large amounts of beer is associated with a markedly less pronounced fat accumulation in the liver when compared to the consumption of plain ethanol, and these effects are widely abolished when beer without hop is applied in a similar model [56]. However, compounds in beer responsible for the less harmful effects on the liver have not yet been identified. Previously, we have shown that xanthohumol, another hop compound, inhibits several critical pathophysiological steps during the development and course of chronic liver disease, including oxidative stress, inflammation, and fibrogenesis [57]. However, this prenylated flavonoid is not heat stable and thus is present in only very low quantities in beer. In contrast, IAA develop during beer brewing, and are heat stable. Therefore, IAA content in beer may eventually explain why it is less harmful for the liver compared to pure alcohol. However, alcohol content in beer certainly surpasses the beneficial IAA effect. Moreover, there is unanimous hesitancy regarding IAA uptake via beer because of the fine line between moderate and binge drinking. However, IAA can also be produced from hop independent of wort boiling during the brewing process. Also the IAA extract used in this study is produced directly from hops, and is applied later during the brewing process. Therefore, it could also be used independent of beer consumption. Still, this extract as well as extracts from other vendors are used since a long time in the brewing industry indicating their safety. Furthermore, IAAs have already been studied in humans with prediabetes and no side effects have been observed [10]. Therefore, it is very promising that the present study newly revealed that IAA inhibit steatosis, inflammation, and fibrogenesis in NAFLD. Yet, it has to be noted that in the present study IAA administration was started at the beginning of the WTD feeding. In future studies, IAA effects should be analyzed after the onset of (liver) disease, which would more closely mirror the potential application of IAA in humans. Nonetheless, our study provides the first indication of the potential of this natural ingredient as novel and safe therapeutic for the prevention and treatment of NAFLD and potentially also in other forms of chronic liver disease.

Acknowledgements This work was supported by a grant from the "Wissenschaftsförderung der Deutschen Brauwirtschaft e.V." (Berlin, Germany) (Project No R437) to $\mathrm{CH}$. $\mathrm{CH}$ and $\mathrm{AB}$ are supported by the German Research Foundation (FOR 2127 and KFO262). We want to thank Rudolf Jung for excellent technical assistance. We acknowledge the Human Tissue and Cell Research (HTCR) Foundation for making human tissue available for research and Hepacult GmbH (Regensburg, Germany) for providing PHH for in vitro studies.

Author contributions $\mathrm{AM}$ and $\mathrm{CH}$ designed the study. $\mathrm{AM}, \mathrm{AK}$ and $\mathrm{KM}$ performed the experiments. TS, WET, CS and $\mathrm{AB}$ provided material. IB and $\mathrm{AB}$ provided technical support. $\mathrm{AM}$ and $\mathrm{CH}$ wrote the manuscript.

\section{Compliance with ethical standards}

Conflict of interest $\mathrm{CH}$ was consultant of Joh. Barth \& Sohn GmbH, and $\mathrm{AM}, \mathrm{AK}$, and $\mathrm{KF}$ are working in the laboratory of $\mathrm{CH}$. $\mathrm{CS}$ is employee of the Joh. Barth \& Sohn GmbH. IB received funding for a research project from Yakult Ltd. All authors had complete and independent control over the study design, analysis and interpretation of data, report writing, and publication, regardless of results. 


\section{References}

1. Dongiovanni P, Anstee QM, Valenti L. Genetic predisposition in NAFLD and NASH: impact on severity of liver disease and response to treatment. Curr Pharm Des. 2013;19:5219-38.

2. Powell EE, Cooksley WG, Hanson R, Searle J, Halliday JW, Powell LW, et al. The natural history of nonalcoholic steatohepatitis: a follow-up study of forty-two patients for up to 21 years. Hepatology. 1990;11:74-80.

3. Mohamad B, Shah V, Onyshchenko M, et al. Characterization of hepatocellular carcinoma (HCC) in non-alcoholic fatty liver disease (NAFLD) patients without cirrhosis. Hepatol Int. 2016;10:632-9.

4. Dietrich P, Hellerbrand C. Non-alcoholic fatty liver disease, obesity and the metabolic syndrome. Best Pract Res Clin Gastroenterol. 2014;28:637-53.

5. Bedossa P. Pathology of non-alcoholic fatty liver disease. Liver Int. 2017;37(Suppl 1):85-89.

6. Hellerbrand C. Hepatic stellate cells-the pericytes in the liver. Pflug Arch. 2013;465:775-8.

7. Mann J, Oakley F, Akiboye F, et al. Regulation of myofibroblast transdifferentiation by DNA methylation and MeCP2: implications for wound healing and fibrogenesis. Cell Death Differ. 2007; 14:275-85.

8. Kunimune T, Shellhammer TH. Foam-stabilizing effects and cling formation patterns of iso-alpha-acids and reduced iso-alpha-acids in lager beer. J Agric Food Chem. 2008;56:8629-34.

9. Minich DM, Lerman RH, Darland G, et al. Hop and acacia phytochemicals decreased lipotoxicity in 3T3-L1 adipocytes, $\mathrm{db} / \mathrm{db}$ mice, and individuals with metabolic syndrome. J Nutr Metab. 2010;2010:467316

10. Obara K, Mizutani M, Hitomi Y, et al. Isohumulones, the bitter component of beer, improve hyperglycemia and decrease body fat in Japanese subjects with prediabetes. Clin Nutr. 2009;28: 278-84.

11. Shimura M, Hasumi A, Minato T, et al. Isohumulones modulate blood lipid status through the activation of PPAR alpha. Biochim Biophys Acta. 2005;1736:51-60.

12. Yajima $\mathrm{H}$, Ikeshima $\mathrm{E}$, Shiraki M, et al. Isohumulones, bitter acids derived from hops, activate both peroxisome proliferator-activated receptor alpha and gamma and reduce insulin resistance. J Biol Chem. 2004;279:33456-62.

13. Everard A, Geurts L, Van Roye M, et al. Tetrahydro iso-alpha acids from hops improve glucose homeostasis and reduce body weight gain and metabolic endotoxemia in high-fat diet-fed mice. PLoS ONE. 2012; 7:e33858.

14. Morimoto-Kobayashi Y, Ohara K, Ashigai H, et al. Matured hop extract reduces body fat in healthy overweight humans: a randomized, double-blind, placebo-controlled parallel group study. Nutr J. 2016;15:25.

15. Namikoshi $\mathrm{T}$, Tomita $\mathrm{N}$, Fujimoto $\mathrm{S}$, et al. Isohumulones derived from hops ameliorate renal injury via an anti-oxidative effect in Dahl salt-sensitive rats. Hypertens Res. 2007;30: $175-84$

16. Minich DM, Bland JS, Katke J, et al. Clinical safety and efficacy of NG440: a novel combination of rho iso-alpha acids from hops, rosemary, and oleanolic acid for inflammatory conditions. Can J Physiol Pharmacol. 2007;85:872-83.

17. Konda VR, Desai A, Darland G, et al. Rho iso-alpha acids from hops inhibit the GSK-3/NF-kappaB pathway and reduce inflammatory markers associated with bone and cartilage degradation. $\mathrm{J}$ Inflamm (Lond). 2009;6:26.

18. Hall AJ, Babish JG, Darland GK, et al. Safety, efficacy and antiinflammatory activity of rho iso-alpha-acids from hops. Phytochemistry. 2008;69:1534-47.
19. Desai A, Konda VR, Darland G, et al. META060 inhibits multiple kinases in the NF-kappaB pathway and suppresses LPS-mediated inflammation in vitro and ex vivo. Inflamm Res. 2009;58:229-34.

20. Ano Y, Dohata A, Taniguchi Y, et al. Iso-alpha-acids, bitter components of beer, prevent inflammation and cognitive decline induced in a mouse model of Alzheimer's disease. J Biol Chem. 2017;292:3720-8.

21. Lee SM, Schelcher C, Laubender RP, et al. An algorithm that predicts the viability and the yield of human hepatocytes isolated from remnant liver pieces obtained from liver resections. PLoS ONE. 2014;9:e107567.

22. Lee SM, Schelcher C, Demmel M, et al. Isolation of human hepatocytes by a two-step collagenase perfusion procedure. J Vis Exp. 2013;79. https://doi.org/10.3791/50615.

23. Hellerbrand, Wang SC, Tsukamoto $\mathrm{H}$. et al. Expression of intracellular adhesion molecule 1 by activated hepatic stellate cells. Hepatology. 1996;24:670-6.

24. Yajima H, Noguchi T, Ikeshima E, et al. Prevention of dietinduced obesity by dietary isomerized hop extract containing isohumulones, in rodents. Int J Obes (Lond). 2005;29:991-7.

25. Starkel P, De Saeger C, Delire B, et al. Tetrahydro iso-alpha acids and hexahydro iso-alpha acids from hops inhibit proliferation of human hepatocarcinoma cell lines and reduce diethylnitrosamine induced liver tumor formation in rats. Nutr Cancer. 2015;67:748-60.

26. Dorn C, Engelmann JC, Saugspier M, et al. Increased expression of c-Jun in nonalcoholic fatty liver disease. Lab Invest. 2014;94:394-408.

27. Wobser H, Dorn C, Weiss TS, et al. Lipid accumulation in hepatocytes induces fibrogenic activation of hepatic stellate cells. Cell Res. 2009;19:996-1005.

28. Mahli A, Thasler WE, Patsenker E, et al. Identification of cytochrome CYP2E1 as critical mediator of synergistic effects of alcohol and cellular lipid accumulation in hepatocytes in vitro. Oncotarget. 2015;6:41464-78.

29. Hellerbrand C, Muhlbauer M, Wallner S, et al. Promoterhypermethylation is causing functional relevant downregulation of methylthioadenosine phosphorylase (MTAP) expression in hepatocellular carcinoma. Carcinogenesis. 2006;27:64-72.

30. Mahli A, Saugspier M, Koch A. et al. ERK activation and autophagy impairment are central mediators of irinotecan-induced steatohepatitis. Gut. 2017;67:746-56.

31. Gabele E, Dostert K, Dorn C, et al. A new model of interactive effects of alcohol and high-fat diet on hepatic fibrosis. Alcohol Clin Exp Res. 2011;35:1361-7.

32. Caballero I, A Blanco C, Porras M. Iso- $\alpha$-acids, bitterness and loss of beer quality during storage. Trends Food Sci Technol. 2012;26:21-30.

33. Rodda LN, Gerostamoulos D, Drummer OH. The stability of iso$\alpha$-acids and reduced iso- $\alpha$-acids in stored blood specimens. Forensic Sci Int. 2014;239:44-9.

34. Arndt S, Wacker E, Dorn C, et al. Enhanced expression of BMP6 inhibits hepatic fibrosis in non-alcoholic fatty liver disease. Gut. 2015;64:973-81.

35. Dorn C, Riener MO, Kirovski G, et al. Expression of fatty acid synthase in nonalcoholic fatty liver disease. Int J Clin Exp Pathol. 2010;3:505-14.

36. Paglialunga S, Dehn CA. Clinical assessment of hepatic de novo lipogenesis in non-alcoholic fatty liver disease. Lipids Health Dis. 2016;15:159.

37. Nakamuta M, Kohjima M, Morizono S, et al. Evaluation of fatty acid metabolism-related gene expression in nonalcoholic fatty liver disease. Int J Mol Med. 2005;16:631-5.

38. Musso G, Cassader M, Gambino R. Non-alcoholic steatohepatitis: emerging molecular targets and therapeutic strategies. Nat Rev Drug Discov. 2016;15:249-74. 
39. Schattenberg JM, Singh R, Wang Y, et al. JNK1 but not JNK2 promotes the development of steatohepatitis in mice. Hepatology. 2006;43:163-72.

40. Tilg H, Moschen AR, Roden M. NAFLD and diabetes mellitus. Nat Rev Gastroenterol Hepatol. 2017;14:32-42.

41. Friedman SL. Mechanisms of hepatic fibrogenesis. Gastroenterology. 2008;134:1655-69.

42. Brenner DA. Molecular pathogenesis of liver fibrosis. Trans Am Clin Climatol Assoc. 2009;120:361-8.

43. Stal P. Liver fibrosis in non-alcoholic fatty liver disease - diagnostic challenge with prognostic significance. World J Gastroenterol. 2015;21:11077-87.

44. Hazra S, Xiong S, Wang J, et al. Peroxisome proliferator-activated receptor gamma induces a phenotypic switch from activated to quiescent hepatic stellate cells. J Biol Chem. 2004;279:11392-401.

45. Marra F, Efsen E, Romanelli RG, et al. Ligands of peroxisome proliferator-activated receptor gamma modulate profibrogenic and proinflammatory actions in hepatic stellate cells. Gastroenterology. 2000;119:466-78.

46. Tilg $\mathrm{H}$, Moschen A. Weight loss: cornerstone in the treatment of non-alcoholic fatty liver disease. Minerva Gastroenterol Dietol. 2010;56:159-67.

47. Sumiyoshi M, Kimura Y. Hop (Humulus lupulus L.) extract inhibits obesity in mice fed a high-fat diet over the long term. Br J Nutr. 2013;109:162-72.

48. Vroegrijk IO, van Diepen JA, van den Berg SA, et al. META060 protects against diet-induced obesity and insulin resistance in a high-fat-diet fed mouse. Nutrition. 2013;29:276-83.
49. Ricci R, Bevilacqua F. The potential role of leptin and adiponectin in obesity: a comparative review. Vet J. 2012;191:292-8.

50. Miura Y, Hosono M, Oyamada C, et al. Dietary isohumulones, the bitter components of beer, raise plasma HDL-cholesterol levels and reduce liver cholesterol and triacylglycerol contents similar to PPARalpha activations in C57BL/6 mice. $\mathrm{Br} \mathrm{J}$ Nutr. 2005;93:559-67.

51. Kirovski G, Dorn C, Huber H, et al. Elevated systemic monocyte chemoattractrant protein-1 in hepatic steatosis without significant hepatic inflammation. Exp Mol Pathol. 2011;91:780-3.

52. Yan H, Gao Y, Zhang Y. Inhibition of JNK suppresses autophagy and attenuates insulin resistance in a rat model of nonalcoholic fatty liver disease. Mol Med Rep. 2017;15:180-6.

53. Nair AB, Jacob $\mathrm{S}$. A simple practice guide for dose conversion between animals and human. J Basic Clin Pharm. 2016;7:27-31.

54. Heyerick A, Zhao Y, Sandra P, et al. Photolysis of hop-derived trans-iso-alpha-acids and trans-tetrahydroiso-alpha-acids: product identification in relation to the lightstruck flavour of beer. Photochem Photobiol Sci. 2003;2:306-14.

55. Kanuri G, Wagnerberger S, Landmann M, et al. Effect of acute beer ingestion on the liver: studies in female mice. Eur $\mathbf{J}$ Nutr. 2015;54:465-74.

56. Landmann M, Sellmann C, Engstler AJ, et al. Hops (Humulus lupulus) content in beer modulates effects of beer on the liver after acute ingestion in female mice. Alcohol Alcohol. 2017;52:48-55.

57. Weiskirchen R, Mahli A, Weiskirchen S, et al. The hop constituent xanthohumol exhibits hepatoprotective effects and inhibits the activation of hepatic stellate cells at different levels. Front Physiol. 2015;6:140. 